International Journal of Psychological Research and Reviews
(ISSN:2639-6041)

\title{
Adolescent Violence: A Narrative Review
}

\author{
Tiffany Field, PhD
}

University of Miami/Miller School of Medicine; Fielding Graduate University

\begin{abstract}
This narrative review is based on a literature search on PsycINFO and PubMed entering the terms adolescent violence for papers published during the last five years. Following exclusion criteria, 58 papers could be classified as school-based violence (fighting) and dating violence including research on the prevalence and risk factors for these types of violence. The prevalence of school violence has varied by ethnicity, type of violence and culture. The risk factors for school violence are both intrapersonal and interpersonal. The intrapersonal factors include male gender, minority status, middle school level, maladaptive cognitive/ emotional strategies, depression, callous behavior, conduct problems, low cortisol and high testosterone, unhealthy conditions including concussions and obesity, drug abuse, self-harm, suicidal ideation and carrying weapons. The interpersonal factors include a lack of parental monitoring, conflict and violence in the family, exposure to violence and to violent social media. The prevalence of dating violence has also varied by gender and culture. The intrapersonal risk factors have included externalizing behavior, sexting both off-line and online, alcohol and marijuana misuse. The interpersonal risk factors include anxious attachment, family violence and peer rejection. Surprisingly, given the prevalence and severity of these problems, very little prevention/intervention research appears in this recent literature. Research is also missing on peer relationships, empathy and psychopathy as potential risk factors. Like other literature on adolescent problems, this research is limited by primarily deriving from self-report, parent report and hospital records.
\end{abstract}

Keywords: adolescent violence, school-based violence, dating violence

*Correspondence to Author:

Tiffany Field, PhD

University of Miami/Miller School of Medicine; Fielding Graduate University

How to cite this article:

Tiffany Field. Adolescent Violence: A Narrative Review. International Journal of Psychological Research and Reviews, 2019, 2:17. DOI: 10.28933/ijprr-2019-06-1605

\section{eScîPub}

eSciPub LLC, Houston, TX USA.

Website: https://escipub.com/ 
This narrative review is based on a literature search on PsycINFO and PubMed entering the terms adolescent violence and publications from 2014-2019. The narrative review is designed to provide a more comprehensive summary of research on adolescent violence than has appeared in the more focused systematic reviews. The years 2014-2019 were selected inasmuch as reviews of the earlier literature had already been published. Exclusion criteria were case studies, pilot studies and non-English papers. And, the criminal justice literature was beyond the scope of this review. Following these criteria, 58 papers published during the last five years (2014-2019) could be classified as school-based violence (fighting) and dating violence including research on the prevalence and risk factors for these types of violence. The prevalence of school violence has varied by ethnicity, type of violence and culture. The risk factors for school violence are both intrapersonal and interpersonal. They include male gender, minority status, middle school level, maladaptive cognitive/emotional strategies, depression, callous behavior, conduct problems, low cortisol and high testosterone, unhealthy conditions including concussions and obesity, drug abuse, self-harm, suicidality and carrying weapons. The interpersonal factors include a lack of parental monitoring, family conflict and violence, exposure to violence and to violent social media. The prevalence of dating violence has also varied by gender and culture. The intrapersonal risk factors have included externalizing behavior, sexting both off-line and online, alcohol and marijuana misuse. The interpersonal risk factors include anxious attachment, family violence and peer rejection. Surprisingly, given the prevalence and severity of these problems, very little prevention/intervention research appears in this recent literature. Recent research is also missing on peer relationships, empathy and psychopathy as potential risk factors, although these risk factors appeared in an older literature. Like studies on other adolescent problems, this research is limited by primarily deriving from self-report and parent report, and hospital records. This narrative review is accordingly divided into sections on research on prevalence and risk factors for school violence followed by similar sections for dating violence. These sections are, then, followed by discussions on methodological limitations of the literature and future directions. Tables are included to simplify the search for specific references on the topics that are reviewed here.

\section{Prevalence of School Violence}

School violence has been defined as fighting including being grabbed, shoved, kicked, punched, hit with a hand or hit with an object (Khoury-Kassabri \& Attar-Schwartz, 2014). The prevalence of school violence has varied by ethnicity, by type of violence and by culture (see table 1). Although the rates of school violence are high, surprisingly, the Centers for Disease Control reported a decrease in the deaths from violence over the years 1999 to 2015 (Ballesteros, Williams, Mack, Simon \& Sleet, 2018). Further, the National Survey on Drug Use and Health revealed a decrease in youth fighting and violence in all racial/ethnic groups over the period of 2002-2014 from a high level of 34\% to a significantly lower level of $24 \%$, suggesting a $29 \%$ decrease in school violence (Salas-Wright, Nelson, Vaughn, Reingle-Gonzalez \& Cordova, 2017).

\section{Ethnicity Differences}

Consistently higher rates of school violence have been noted for African-American adolescents, followed by Hispanic and then nonHispanic white adolescents, possibly related to socioeconomic factors and school locations (Salas-Wright et al, 2017). However, reductions were reported for Hispanic adolescent violence in the Youth Risk Behavior Survey from 20012015 (Khubchandani \& Price, 2018). This survey specifically focused on the prevalence of violence-related behavior and weapon-carrying by Hispanic adolescents. The females showed a reduction in fighting and fighting on school property, while the males not only showed a

IJPRR:https://escipub.com/international-journal-of-psychological-research-and-reviews/ 2 
reduction in physical fights and physical fighting on school property but also fewer threats with weapons.

\section{Violence-Related Injuries}

Despite these reduced rates of fighting, the National Electronic Injury Surveillance System showed that overall rates of nonfatal violencerelated injuries among adolescents remained stable across at least the years 2009 to 2013 (Bell, Qiao, Jenkins, Siedlecki \& Fecher, 2016).
Violence-related problems are also reflected in the number of emergency admissions for violence-related injury, reportedly at $4 \%$ for adolescents (Herbert, Gilbert, GonzalezIzuierdo \& Li, 2015). Recurrent emergency admissions occurred more often for adversityrelated injuries than for accident-related injuries. These injuries accounted for $50 \%$ of all the emergency admissions for girls and $29 \%$ for boys.

Table 1. Prevalence of school violence.

\begin{tabular}{lll}
\hline Prevalence & Country & First Author \\
\hline $24 \%$ & U.S. & Salas-Wright \\
$11 \%$ & Ontario & Ilie \\
$24 \%$ & Holland & Yu \\
$36 \%$ & Peru & Sharma \\
$33 \%$ & India & Swain \\
$37 \%$ & Pakistan & Rao \\
$44 \%$ & Sri Lanka & Senanayake \\
$49 \%$ & Qatar & Bala \\
$30-66 \%$ & Iran & Sadeghi \\
$56 \%$ & Israel & Khoury-Kassabri
\end{tabular}

\section{Verbal Versus Physical Violence}

Some researchers have made the distinction between verbal and physical violence in adolescents. In one study, the prevalence of verbal violence was $46 \%$ while the prevalence of physical violence was significantly lower at 33\% (Golshiri, Farajzadegan, Tavakoli \& Heidari, 2018). This database included middle school and high school students. In an exclusively middle school sample, the rate of verbal violence was similarly high (at $49 \%$ ), although only $7 \%$ of students were involved in physical fights (Bao, Jing, Yang, Li \& Cai, 2016). Nonetheless, in this large sample ( $\mathrm{N}=3315$ students), as many as $2 \%$ were injured in fights.

\section{Cultural Differences}

The adolescent violence rates differ in different cultures. Although cross-cultural comparisons have not been made in this recent literature on adolescent violence, the following examples of prevalence in different cultures are presented in the order of increasing violence.

In Ontario, relatively low prevalence rates have been noted including $11 \%$ for fighting, $6 \%$ for beating up or hurting someone and $6 \%$ for carrying a weapon (gun or knife) on school property (llie, Mann, Boak, Hamilton, Rehm, et al, 2017). ( $\mathrm{N}=5478)$. In a Dutch sample of adolescents $(\mathrm{N}=358)$, as many as $24 \%$ of adolescents showed violent behavior ( $\mathrm{Yu}$, Branje, Meeus, Cowen, \& Fazel, 2018). In a cross-sectional study of 1368 randomly selected Peruvian adolescents, an even higher rate of students $(36 \%)$ had been involved in fighting during the last year (Sharma, Nam, Kim \& Kim). In a sample from southern India, a similarly high prevalence of fighting was noted at $33 \%$ for one incident of violence and $18 \%$ for at least two incidents (Swain, Mohanan, Sanah, Sharma, \& Ghosh, 2014). The incidence of serious fights was less at $11 \%$, while as many as 
$22 \%$ thought about hitting somebody, and $16 \%$ of boys and $10 \%$ of girls carried sharp objects to school.

A national survey of 4583 Pakistani adolescents revealed a relatively high prevalence of $37 \%$ for adolescents aged 13 to 15 years being in physical fights (Rao, Shah, Jawed, Inam \& Shafique, 2015). An even higher rate (44\%) was reported for fighting one or more times in a sample of Sri Lankan adolescents $(\mathrm{N}=3262)$ (Senanayake, Gunawardena, Wickramasinghe, Wickramasinghe, Gunawardena,et al, 2019). A similarly high rate has been noted for the reputedly "most advanced Arab country" (Qatar) at $49 \%$ (Bala, Chehab, Al-Dahshan, Saadeh, \& Al Khenji, 2018). The rate of violence in an Iranian sample of adolescents was given as ranging from 30 to $66 \%$ (Sadeghi, Farajzadegen, Kelishadi \& Heidari, 2014). And, in a sample of 1324 Jewish and Arab adolescents living in northern Israel, $56 \%$ of adolescents surveyed reported at least one form of physical violence, which may reflect the larger ongoing conflict in that country (Khoury-Kassabri et al, 2014).

The prevalence of physical violence appears to be greater when counting victims of violence. For example, $76 \%$ of Mexican adolescents have been reputedly victims of physical aggression in their relationships, with $16 \%$ having experienced physical violence and $17 \%$ of girls having been the victims of sexual violence (Sosa-Rubi, Saavedra-Avendano, Piras, Van Buren \& Bautista-Arredondo, 2017). In the same sample, female adolescents were noted to perpetrate physical violence more frequently than males.

The highly variable prevalence rates for these cultures may relate to the studies being highly variable on their sources of their information (e.g. self-report, parent report, and medical records), in the nature of their surveys, their sample sizes, the age groups they have surveyed, the definition of violence, the measure of violence and the years covered by the surveys. In addition, socioeconomic differences and education system differences may contribute to their variability. A cross-cultural comparison would control for the variability on these factors. In addition, cross-cultural comparisons might more reliably identify predictor variables that contribute to this variance across cultures. That would, in turn, potentially inform prevention/intervention programs.

\section{Risk Factors for School Violence}

Many risk factors have been identified for adolescent school violence. These have been categorized here as intrapersonal and interpersonal factors.

\section{Intrapersonal Risk Factors}

Many intrapersonal risk factors for school violence have appeared in the recent literature (see table 2). They include being male gender, minority status, being younger middle-school age adolescents, having maladaptive cognitive/emotional strategies, being depressed, having low cortisol and elevated testosterone, being callous and having conduct problems, experiencing unhealthy conditions, drug abuse, self-harm and suicidal ideation and carrying weapons.

Gender. Being male gender has been a significant risk factor for adolescent school violence in several samples in many countries in different parts of the world. These include two samples in Iran (Golshiri et al, 2018; Sadeghi et al, 2014). In the latter study, the prevalence of adolescent violence in males was 2.5 times the rate of violence in females. In other samples, male gender was a significant risk factor including a sample in Sri Lanka (Senanayake et al, 2019), in rural Delhi (Anand, Kishore, Grover, Bhave \& Yadav, 2018) and among Arab-Israeli adolescents in northern Israel schools (Agbaria \& Daher, 2015). And, in a study in this recent literature, more females between the age of 15 and 19 were the victims of violence (deOliveira, Pinto, de Maacedo, de Oliviera \& Cavalcanti, 2014). In this Brazilian sample, $57 \%$ of females and $43 \%$ of males were victims of violence based on medical and police reports. Most of the 
violence occurred on public streets (56\%) 2015) as well as low cortisol levels (Yu et al, followed by the home (33\%), and the most 2018) which is reputedly a biochemical profile for affected body region was the face (44\%), aggression. These two measures would need to variables that are rarely studied. The greater be assessed in one study to document the high prevalence for male violence may be explained testosterone-low cortisol profile and its by elevated testosterone (Peckins \& Susman, relationship to adolescent violence.

Table 2. Intrapersonal risk factors for school violence.

\begin{tabular}{ll}
\hline Risk Factor & First Author \\
\hline Male gender & Golshiri, Sadeghi, Senanayake, \\
Non-Hispanic & Anand, Agbaria \\
African-American & Salas-Wright \\
First generation immigrants & Pottie \\
Middle school students & Golshiri \\
Younger males & Senanyake, Bao \\
Maladaptive emotion regulation (catastrophizing) & Bao \\
Negative self-perception health & Silva \\
Physical inactivity, fast food & Rao \\
Overweight & Stensland \\
Concussions & Buckley \\
Depression & Yu \\
Low waking cortisol activity & Yu \\
Elevated testosterone & Peckins \\
Callous-unemotional traits & Baskin-Sommers \\
Externalizing problems & Mrug \\
Smoking & Rao, Kelishadi, Senanayake \\
Alcohol & Calvete, Senanayake, Herbert \\
Self-harm & Bell \\
Suicidal thoughts \& attempts & Silva, Senanyake \\
Carrying weapons & Golshiri, llie, Khubchandani \\
\hline
\end{tabular}

Minority status. Adolescents who have minority status related to their ethnicity or their being immigrants have notably higher rates of fighting and violence. At least one study has documented a greater prevalence of fighting and violence among African-American adolescents (Salas-Wright et al, 2017). This estimate, based on data from the National Survey on Drug Use and Health, was higher than the prevalence of violence among Hispanic adolescents and the lowest prevalence was for non-Hispanic white adolescents. In a systematic review of 18 studies on immigrant adolescents' violence, firstgeneration immigrant adolescents showed greater rates of peer aggression than thirdgeneration and native adolescents (Pottie, Dahal, Georgiades, Premji \& Hassan, 2015).

Age/grade level. A comparison between middle school and high school students surprisingly suggested a greater prevalence of physical violence among middle school students than high school students (Golshiri et al, 2018). In a similar study in Sri Lanka, violence was more prevalent in the 13 to 15 -year-old males 
than in the 16 and 17-year-old males (Senanayake et al, 2019). Greater fighting among the younger males may relate to their less developed socialization and self-regulation. It could also relate to a reactive aggression/defensive aggression due to their lesser size at that age. Surprisingly, aggression in these studies has not been classified as proactive or reactive aggression.

\section{Maladaptive cognitive emotion regulation} strategies. In a large sample study $(\mathrm{N}=3315)$ in which middle school boys again were more often involved in fights, the adolescents completed anonymous, self-report questionnaires on their cognitive emotion and violence-related behaviors (Bao et al, 2016). A logistic regression on these data suggested that six cognitive emotion strategies were related to violent behavior including blaming others, catastrophizing, reappraisal, planning, rumination and self-blame. Catastrophizing was the factor that was most associated with violent behavior.

Unhealthy conditions. Unhealthy conditions that have contributed to violent behavior in adolescents have appeared in the recent literature. These include unhealthy lifestyle habits, being overweight and physical trauma, i.e. concussions. In a study on Brazilian adolescents' self-perceptions of health $(\mathrm{N}=2614), 27 \%$ of adolescents had a negative self-perception of health (Silva, Silva, Passos, Soares, Valenca et al, 2018). This more commonly occurred in girls. In a multivariate study on the Global School Health Survey in Pakistan, unhealthy behaviors including physical inactivity, smoking, soft drinks and fast food occurred in $39 \%$ of the 13 to 15-year-old adolescents in the sample in which $37 \%$ had been involved in physical fighting (Rao et al, 2015). The students who had four or more of these unhealthy behaviors were more likely to be involved in physical fighting. Given the prevalence of physical inactivity and consumption of fast food in this sample, it is not surprising that in an adolescent violence study in
Norway, a significant relationship was noted between interpersonal violence and body mass index after adjusting for unfavorable lifestyle, socioeconomic and psychosocial factors in a multiple regression analysis (Stensland, Thoresen, Wentzel-Larsen \& Dyb, 2015).

Physical trauma has also been associated with adolescent violence. For example, in a survey on Australian adolescents from 13 high schools, $13 \%$ of adolescents reported that they had a concussion (Buckley \& Chapman, 2017). In a logistic regression model, concussion at the baseline assessment period predicted violent behavior (getting in a fight) one year later. It is not clear, however, whether the adolescents' concussions were related to earlier fighting, suggesting that the predisposition to violence contributed to the baseline concussions as well as to the later violent behavior.

\section{Depression and related hormones.}

Depression has been associated with adolescent violence. However, it is not clear whether depression precedes, results from, or is comorbid with adolescent violence because most of the findings on this association are based on cross-sectional data. As an exception, a longitudinal database from Holland suggested that depression preceded adolescent violence (Yu et al, 2018). In this study, $24 \%$ of adolescents perpetrated violence. In a regression analysis, the cortisol awakening response (waking cortisol activity) moderated the effects of depressive symptoms on later violent behavior. Specifically, when the cortisol awakening response was low, depressive symptoms were associated with later violent behavior. Elevated testosterone and low cortisol levels have been associated with aggressive behavior in earlier studies (Diego, Field, Hernandez-Reif, Shaw, Castellanos \& Roth, 2002). And, in another study, testosterone levels were related to adolescent violence (Peckins et al, 2015). However, cortisol was not assayed in this study, so the association between elevated testosterone, low cortisol and violence could not be assessed. 
Callous-unemotional traits and conduct problems. Surprisingly, only two studies could be found in the recent adolescent violence literature on conduct problems. In one study on male adolescents, callous-unemotional traits in the presence of conduct problems contributed to violence and substance abuse after controlling for a number of other environmental risks (Baskin-Sommers, Waller, Fish, \& Hyde, 2015). In a structural equation modeling study on a community sample of adolescents, more externalizing problems (anti-social, maladaptive behaviors directed toward others) at age 13 predicted more violent behavior at age 18 (Mrug, Madan \& Windle, 2016). Although this was not a surprising result, it is unfortunate that this was the only longitudinal study in the recent literature that assessed externalizing behavior.

Substance abuse. Smoking and alcohol are the two most frequent substance abuse problems associated with adolescent violence in this recent literature. In a large sample, Iranian adolescents $(N=13,486)$ living in rural and urban areas were selected via a cluster sampling method and given a questionnaire on psychiatric distress, violence behaviors and smoking status (Kelishadi, Babaki, Qorbani, Ahadi, Heshmat et al, 2015). Both psychiatric distress and violent behaviors were positively associated with smoking status. Both smoking and alcohol use were associated with fighting with peers in a study on adolescents in Sri Lanka (Senanayake et al, 2019). And, in a large sample of adolescents in England ( $N=141,248)$, hospital admissions for drug or alcohol misuse commonly occurred in the same girls and boys who were admitted for injuries related to violence (Herbert et al, 2015). In an unusual child-to-parent violence study, substance use at baseline predicted physical aggression against parents 12 months later (Calvete, Orue\& GamezGuadix, 2015). This association was significant only for boys.

Self-harm and suicidal ideation. Although the violence-related injuries among adolescents have not changed significantly according to a recent US survey, the self-harm injury rates have significantly increased for female, younger adolescents during the same period (Bell et al, 2016). Suicidal thoughts have also been associated with school violence in a study on Brazilian adolescents (Silva et al, 2018). Both thoughts and attempts have been associated with adolescent violence in the Sri Lanka Global School-Based Health Survey (Senanayake et al, 2019). Because violence and suicidal thoughts were reported contemporaneously, it is not clear whether suicidal thoughts preceded or followed adolescent violence. At least one study suggests that violence experienced at home, at school and in the community led to suicidal ideas based on an analysis of descriptions given by Vietnamese adolescents (Hong Le, Tran, Nguyen \& Fisher, 2018).

Carrying Weapons. Carrying weapons and threatening school violence have been associated with physical violence in a study from Iran (Golshiri et al, 2018). In this study, being a boy, carrying a weapon out of home or in school and violence in the home were significant risk factors for physical violence. Data on adolescents from Ontario suggested that as many as $6 \%$ reported carrying a weapon (e.g. gun or knife) on school property (llie et al, 2017). Although violence and weapon carrying are usually assessed during the same time period, adolescent violence as well as drug use were highly predictive of weapon carrying based on 2001-2015 longitudinal data from the Youth Risk Behavior Survey (Khubchandani et al, 2018). In a study on "adolescent copycats" who threatened to carry out a school massacre following two Finnish school shootings, the copycats were noted to show symptoms of anxiety, depression and suicidal ideation as well as non-physical and physical violence against others (Lindberg, Sailas \& Kaltiala-Heino, 2012).

\section{Interpersonal Risk Factors}

Several interpersonal risk factors could also be found in the recent literature on adolescent violence (see table 3). These are categorized 
here as lack of parental monitoring, family conflict/violence, exposure to violence and exposure to violent social media and networking.

Lack of parental monitoring. In the Sri Lanka study that featured a high rate of adolescent fighting with peers $(44 \%)$, parents "not being aware of students' activities" was associated with adolescent violence (Senanayake et al, 2019). And, in a U. S. study, parental monitoring was a significant protective factor against adolescent violence even after accounting for other risk factors (Khurana, Bleakley, Ellithorpe, Hennessy, Jamieson et al, 2019). In contrast, and surprisingly, parental supervision was positively associated with physical fighting in a study on violence among adolescents in Qatar (Bala, Chehab, Al-Dahshan, Saadeh \& Al Khenji, 2018). This may have related to restrictive/authoritarian parenting styles that are known to have negative effects.

\section{Table 3. Interpersonal risk factors for school violence}

\begin{tabular}{ll}
\hline Risk Factor & First Author \\
\hline Lack of parental monitoring & Senanyake, Khurana \\
Parental supervision & Bala \\
Family conflict & Khurana \\
Domestic violence & Kulaki-Altintas, Golshiri, Sharma \\
School violence exposure & Mrug, Farrell, AlBuhairan, Kim, Wall Meyers \\
Social media and social network violence exposure & Khurana, Bond
\end{tabular}

Family conflict/violence. Family conflict has been a significant predictor of adolescent violence in the U.S. (Khurana et al, 2019). And, high school students who have experienced domestic violence have also shown a greater tendency for violence (Kulakci-Altintas \& AyazAlkaya, 2019). In a similar cross-sectional study on adolescents in Iran, violence in the family was a significant predictor of student violence (Golshiri et al, 2018). And, in a cross-sectional study on 1368 Peruvian adolescents, as many as $39 \%$ of adolescents witnessed violence against their mother and $28 \%$ of adolescents witnessed violence against their father (Sharma, Nam, Kim, \& Kim, 2016).

Exposure to school violence. Exposure to violence across multiple contexts/settings including home, school and community has been researched in a longitudinal study on adolescents age 11, 13 and 18 years (Mrug et al, 2016). In a structural equation model in that study, exposure to high levels of violence at age 11 as well as exposure to violence across multiple contexts were associated with lower levels of internalizing problems (negativity that is focused inward). And, in turn, fewer internalizing problems and more externalizing problems at age 13 predicted more violent behavior at age 18. In another longitudinal study on 1156 sixthgraders, witnessing violence and engaging in physical aggression had bidirectional longitudinal effects on each other (Farrell, Mehari, Kramer-Kuhn \& Goncy, 2014).

Exposure to physical violence at school has been a prevalent problem in several cultures. For example, in a study from Saudi Arabia, 33\% of adolescents reported being exposed to physical violence at school during the past year (AlBuhairan, Abou Abbas, El Sayed, Badri, Alshahri et al, 2017). Those who were exposed to physical violence had a greater odds of having poor academic performance as well as depression and anxiety symptoms. School violence and depression were also associated with each other in a study on adolescents in South Korea (Kim, Kim \& Kim, 2016). And, violence exposure had direct effects on both reactive and proactive aggression 18 months later in a longitudinal study on a sample of 1216 first-time juvenile offenders (Wall Myers, 
Salcedo, Frick, Ray, Thornton et al, 2018). The relationship between violence exposure and proactive aggression was not significant after controlling for baseline proactive aggression and its interaction with reactive aggression. However, violence exposure predicted reactive aggression at the later stage even when baseline reactive aggression and its interaction with proactive aggression were controlled for in the data analysis. The recent research on adolescent violence has typically not distinguished reactive and proactive aggression/violence, although those two types of violence may have very different underlying mechanisms and risk factors.

\section{Exposure to social media and social network} violence. Social media violence is also a risk factor for adolescent violence. Given the significant increase in adolescent Internet addiction (Field, 2018a), it is not surprising that social media is a risk factor for adolescent violence. Adolescent cyberbullying (Field, 2018b), depression (Field, 2019a) and suicide ideation(Field, 2019b) are also increasingly common risk factors. In a recent survey on adolescent $(\mathrm{N}=1990)$ violence, data were content analyzed on top-grossing films and popular TV shows in the U.S. (Khurana et al, 2019). Exposure to media violence was one of the strongest predictors of adolescent aggression after impulsivity and family conflict.

The contagious spread of violence through social networks has been assessed in a sample of adolescents (N=90118) in the National Longitudinal Study of Adolescent Health (Bond \& Bushman, 2017). In this self-report study, the influence of social networks was suggested by the adolescents being $48 \%$ more likely to get in a serious fight, $183 \%$ more likely to have hurt someone badly and $140 \%$ more likely to have pulled a weapon on someone if a friend had engaged in the same behavior. That influence was noted to be up to 4 degrees of separation (i.e. a friend of a friend of a friend of a friend) for serious fights, 2 degrees for hurting someone badly and 3 degrees of separation for pulling a weapon on someone. Given the data from these studies, it is surprising that so little recent research has been conducted on social media and social networking effects on adolescent violence.

\section{Prevalence of Dating Violence}

Dating violence is the other major issue in the recent literature on adolescent violence. It has been variously called inter-partner violence, intimate partner violence, relationship violence and sexual violence (Sosa-Rubi et al, 2017). Dating violence has been further classified as psychological, physical and sexual. In a large sample study ( $\mathrm{N}=1454$ adolescents), a significant association was noted between relationship violence and school violence as well as carrying a weapon among both males and females (DuPont-Reyes, Fry, Rickert, Bell, Palmetto et al, 2014). Most of the findings in this literature are based on adolescent responses to a few self-report scales including the Attitudes Toward Dating Violence Scale (Courtain \& Glowacz, 2018), the Violence in Adolescents' Dating Relationships Inventory and the Conflict in Adolescent Dating Relationships Inventory which have convergent validity (Aizpitarte, Alonso-Arbiol \& Van de Vijver, 2017a). Like school violence, the prevalence of dating violence has varied by gender and by culture (Haglund Belknap, Edwards, Tassara, Joven et al, 2018).

Although cultural differences might be expected for the prevalence of dating violence, crosscultural comparison studies have not been conducted. The prevalence reported for single cultures has ranged from $3 \%$ to $14 \%$ across different ethnic groups within the US and across different countries (see table 4). A couple examples are given here in the order of increasing prevalence. In a large sample of middle and high school students who responded to the California Healthy Kids Survey $(\mathrm{N}=85,198), \quad 10 \%$ of adolescents reported experiencing physical dating violence (Loeb, Deardorff \& Lahiff, 2014). A wider range of prevalence has been reported in a study from 
South Africa (Pollanen et al, 2018). In that study on adolescents from 42 randomly selected high schools $(\mathrm{N}=2199)$, violence ranged from $3 \%$ to $11 \%$, with boys more often being the perpetrators.

\section{Risk Factors for Dating Violence}

As for school violence, dating violence risk factors can also be classified as intrapersonal or interpersonal. This arbitrary classification is based on whether it is an individual characteristic or one that relates to social interaction.

Table 4. Prevalence and risk factors for dating violence.

\begin{tabular}{lll}
\hline Prevalence & Location & \multicolumn{1}{c}{ First Author } \\
$10 \%$ & California & \multicolumn{1}{c}{ Loeb } \\
$11 \%$ & Sollanen & \multicolumn{1}{c}{ First Author } \\
Intrapersonal Risk Factors & & Pollanen \\
Male gender & Hautala, Sosa-Rubi \\
Female gender & Hong Le \\
Females $>$ physical violence & Barreira, Courtain \\
Males $>$ relational violence & Ohlert \\
Externalizing & Morelli \\
Sexting & Singh, Russell \\
Alcohol misuse & Johnson \\
Marijuana use & First Authors \\
Interpersonal Risk Factors & Aizpitarte \\
Anxious attachment & Hong Le \\
Family violence & Baker \\
Peer rejection &
\end{tabular}

\section{Intrapersonal risk factors}

The dating violence intrapersonal risk factors are somewhat different from those for school violence. In this recent literature, the intrapersonal risk factors include gender, externalizing behavior, sexting, alcohol misuse and the use of marijuana.

Gender. Like school violence, at least one study has suggested that boys are more frequently the perpetrators of sexual violence ( $11 \%$ versus $3 \%)$ (Pollanen, de Vries, Mathews, Schneider \& de Vries, 2018). However, at least two other studies have noted females to be more frequently the perpetrators. In one of these studies on a sample of indigenous adolescents from the upper-Midwest of the US and Canada, females were more likely to report being perpetrators only while males were more likely to report being victims only (Hautala, Sittner-Hartshorn,
Armenta \& Whitbeck, 2017). Similarly, in a sample from Mexico City, females were noted to perpetrate dating violence more often than males (Sosa-Rubi et al, 2017).

Within intimate relationships, the type of dating violence appears to differ by gender. Partners attack each other both physically and psychologically, suggesting a pattern of bidirectionality (Barreira, de Lima, Bigras, Njaine \& Assis, 2014). In this study on adolescents in Brazil $(\mathrm{N}=355)$, violence was bidirectional on most forms studied (84\%). Girls reported greater levels of perpetration of physical violence and boys reported more perpetration of relational violence. In a larger sample $(\mathrm{N}=1014)$, once again, the physical violence perpetration rate was higher for females and the relational and sexual violence perpetration rates were higher for males (Courtain et al, 2018). In this study, 
emotional violence was also more frequently perpetrated by females, and, physical victimization occurred more frequently for males and sexual victimization for females. Males and younger adolescents also showed greater tolerance for every form of dating violence. With respect to their expressed attitudes, the adolescents were more tolerant of maleperpetrated psychological violence and more tolerant toward female-perpetrated physical and sexual violence. This study suggests multiple patterns of relationships between perpetration, victimization and tolerance which have rarely been explored together in this literature. In still another culture (Viet Nam), verbal, physical and sexual intimate partner violence were more common for females than males (Hong Le et al, 2014). More males reported being controlled by their female partners. Although the authors related this to more exposure to childhood sexual abuse among young females versus young males, sexual abuse has not been explored as a risk factor for adolescent violence in this recent literature

Externalizing behavior. Perpetrators of sexual violence show more externalizing behavior unlike victims of violence who show more internalizing behavior (Ohlert, Seidler, Rau, Fegert \& Allroggen, 2017). These findings are based on a sample of adolescents living in residential care who completed the Youth Self Report on their lifetime experience with sexual violence (both as perpetrator and victim). Victimperpetrators showed more externalizing behavior. In that respect, they behaved more like perpetrators than like victims.

Sexting. Sexting (i.e. sending sexually explicit or provocative text messages, photos and videos via Internet, smartphone or social networks) has been related to dating violence in adolescents (Morelli, Bianchi, Baiocco, Pezzuti \& Chirumbolo, 2016). In this study, 1334 adolescents completed the Kinsey Scale, the Sexting Behavior Questionnaire, and the Conflict in Adolescent Dating Relationships Inventory. Males and non-heterosexuals engaged in more sexting. And, the high/moderate sexting adolescents committed more off-line and online dating violence. Surprisingly, this is the only research that could be found in the recent literature on sexting and dating violence despite the increasing prevalence of both problems in adolescents.

Alcohol misuse. In a large sample $(\mathrm{N}=842)$ of adolescents seeking emergency room care for alcohol misuse, as many as 22\% reported dating violence in the past year (Singh, Epstein-Ngo, Cunningham, Stoddard, Chermack et al, 2015). The significant correlates of dating violence in this study not only included alcohol use severity but also female gender, suicidal ideation and peer influences. In a sample of very young adolescents (eighth grade students) from Cape Town, South Africa ( $\mathrm{N}=549)$, the majority of adolescents $(79 \%)$ had a partner and reported high rates of intimate partner violence. (Russell, Cupp, Jewkes, Gevers \& Mathews) Over $10 \%$ of boys were perpetrators and $39 \%$ of girls were victims in this sample. The effect of heavy alcohol drinking on intimate partner violence was mediated by the use of negative conflict resolution styles including walking off angrily, sending angry text messages or refusing to talk with a partner. These negative conflict-resolving styles were more notable among the eighth grade girls than boys.

Marijuana use. In one of the only reviews and meta-analyses appearing in this recent literature, 13 articles for the years 2003-2015 were found on the association between marijuana and physical dating violence (Johnson, LaValley, Schneider, Musci, Pettoruto et al, 2017). Of these 13 articles, 11 addressed perpetration and 5 addressed victimization. The authors reported that marijuana use was associated with a $45 \%$ increase in the odds of perpetration and a $54 \%$ increase in the odds of victimization. Once again, the association of this risk factor with dating violence was stronger for girls than boys and for younger versus older adolescents.

\section{Interpersonal Risk Factors}


Just as for school violence, interpersonal risk factors for dating violence appear in the recent literature. These include anxious attachment, family violence and peer rejection.

Anxious attachment. Anxious attachment as a dating violence risk factor was explored in a sample of students $(\mathrm{N}=477)$ in Spain (Aizpitarte, Alonso-Arbiol, Van de Vijver, Perdomo, GalvezSorbal et al, 2017b). Using a structural equation model, the relation between anxious attachment and dating violence was partially mediated by aggressiveness. And, perceived peer dating violence was a direct predictor of dating violence. Further, inter-parental conflict resolution was associated with dating violence that was, in turn, mediated by aggressiveness.

Family violence. Family violence has been a notable risk factor for dating violence just as it has been a risk factor for school violence (Hong Le et al, 2014). That family violence would be a risk factor for both dating and school violence is not surprising. However, this sample of Vietnamese couples who were married before age 18 may not be representative. In addition, in this sample, both early marriage and all forms of intimate partner violence (verbal, physical and sexual) were more common among females than males. More males than females reported being controlled by their partner.

Peer rejection. Peers have also played a role in the dating violence history of adolescents
(Baker, 2017). In this study, eight same-gender focus groups of high school adolescents discussed their prior relationship problems. The adolescents described how "peers help them initiate dating relationships, but once in the relationship, peers would spread rumors and create dramas that led to jealousy, discord and violence between the couple."

\section{Prevention/intervention rograms}

Although no studies could be found in the recent literature on prevention/ intervention for school violence, several types of interventions have focused on dating violence (see table 5). Seeking help from friends and religiosity have been protective factors for preventing dating violence. In a review of the recent literature, 19 studies suggested that adolescents are more likely to go to informal sources of support, with friends being the most commonly reported source of support (Bundock, Chan \& Hewitt, 2018). Females were more likely to seek help than males. Adolescents in these studies identified a number of barriers for seeking help for dating violence, most especially emotional factors, although very little research was found on that topic. In a study based on the National Survey on Drug Use and Health (2006-2010) sample $(N=90,202)$, religiosity was associated with a lesser likelihood of fighting and violent attacks (Salas-Wright, Vaughn \& Maynard, 2014).

\section{Table 5. Prevention intervention programs for dating violence}

\begin{tabular}{ll}
\hline Program & First Author \\
\hline Friends as support & Bundock \\
Religiosity & Salas-Wright \\
Longer term repeated curricula/variety of settings & Lundgren \\
Classroom-based curricula & Sosa-Robi, Gonzalez-Guarda \\
Narrative therapy & Draucker \\
\hline
\end{tabular}

A number of prevention/intervention programs have been tried including classroom, school, community and parenting programs. In addition, other therapeutic efforts have included seeking help from friends and writing narratives about violence issues. In a general review of the literature, 61 interventions were found including 16 community-based, 31 school-based and eight parenting interventions (Lundgren \& Amin, 2015). All of these focused on interventions that 
addressed violence perpetrated by boys against girls in heterosexual relationships. The programs that were the most effective were longer term programs that repeated curricula in a variety of settings rather than awareness raising or discussion sessions. Methodological problems with the studies reviewed included weak research designs, unreported retention rates and short follow-up periods.

Classroom-based curricula have been compared to school climate change programs (Sosa-Rubi et al, 2017). In this comparison, 885 Mexican students were exposed to a classroombased curriculum and 542 to a school climate component only. The 16-week curriculum focused on gender roles, dating violence, strategies for coping with dating violence and sexual rights. A $58 \%$ greater reduction was noted in the prevalence of perpetration of psychological violence. A similar curriculum for the prevention of dating violence in Hispanic adolescents had medium to strong effects on dating violence perpetration for male students but not for females (Gonzalez-Guarda, Guerra, Cummings, Pino, \& Becerra, 2015). And, the intervention effects were not statistically significant at a 12 month follow-up.

Narrative therapy is another approach for reducing dating violence. This therapy, based on identifying unique outcomes in problem-oriented narratives, has appeared in at least one study on adolescent dating violence (Draucker, Smith, Mazurczyk, Thomas, Ramirez et al, 2016). In this study, topics that were focused on big outcomes were extracted from transcripts of interviews with 88 adolescents who were involved in dating violence. The six unique outcomes identified were stories on changing it up, getting back on track, cutting loose, cutting off, standing up for myself, and facing facts.

\section{Limitations and Future Directions}

Several limitations can be noted for this recent literature. The prevalence studies suggest a wide variation of violence rates across different countries. This may relate to violence being defined differently in different countries, the use of different measures, different age samples and different types of data analyses. Cross-cultural comparison studies would help inform not only risk profiles but also intervention efforts.

Although the samples for most of the risk studies were very large, the data sets were limited to very few risk factors and sometimes focused on only one variable. Given the large samples, it is surprising that so few variables were assessed. Multivariate models that could be tested by structural equations, moderating/mediating, profile or multiple regression analyses would seemingly yield more information on profiles of adolescents at risk for becoming violent. Several intrapersonal risk factors like loneliness, lack of empathy, anger and psychopathy were not assessed in recent studies. In addition, several interpersonal risk factors were not measured including child abuse, marginalization as a minority group, parent relations, peer relations, popularity, school attendance, school activities, and academic performance.

The relationship between the lack of physical affection and verbal and physical aggression in an older study highlights the importance of studying physical affection in family relationships and peer relationships as potential factors contributing to violence (Field, 1999). In that study, adolescents in Paris were more physically affectionate toward each other (i.e. showed more hugging and touching each other) than adolescents in Miami who showed more selftouching. The French adolescents were, in turn, less verbally and physically aggressive. Related relationship variables like heartbreak and breakup distress which are reportedly common problems presented to school counselors have also been missing from this recent literature (Field, 2009). The dating violence literature in particular is suggestive of the need for more in depth study of romantic relationships.

The influence of social media and social networking on violence highlights the need for exploring relationships between Internet addiction (Field, 2018a), cyberbullying (Field, 
2018b) and violence. These are likely comorbid problems. Other problems like anxiety, depression and suicidal ideation have been comorbid with both the violence and substance abuse that has been prevalent in adolescents (Field, 2019a, 2019b). But these comorbidities are not usually assessed in the same studies. And, directionality has rarely been determined, as most of the studies are cross-sectional rather than longitudinal. The direction of effects is questionable given that many of the risk factors for violence could also be considered effects of violence. For example, school fighting could lead to concussions as much as concussions have been considered a risk factor for violence. Longitudinal multivariate studies are needed to provide a more comprehensive profile of adolescents at risk for violent behavior. Most of the studies have been cross-sectional instead of longitudinal, limiting any inferences about causality.

Several of the studies on the prevalence and risk of violence in adolescents have relied on school and hospital records rather than assessing the violent adolescents themselves. And those that have focused on the violent adolescents themselves have relied on self-report measures that have questionable anonymity given that many were taken at school. The students may have been under-reporting or "faking good" to avoid embarrassment or shame related to their violent behavior. Thus the data would have questionable generalizability. In addition, the self-reported risk factors were not always measured by psychometrically sound instruments. The surveys/questionnaires were often short and simple in the interests of student compliance. Longitudinal multivariate studies are needed to provide a more comprehensive profile of adolescents at risk for violent behavior. These might include parent, teacher, sibling and peer reports as well as in-depth qualitative interviews, observational and laboratory studies of those adolescents who have engaged in violent behavior.
In an earlier review of the literature on violence in adolescents, empirical research was cited suggesting a model for behavioral, central nervous system and neurotransmitter/neurohormone dysregulation in violent adolescents (Field, 2002). In this model, an underaroused central nervous system was manifested by right frontal EEG hypoactivation. In addition, a neurotransmitter/neurohormone profile was suggested including elevated dopamine and testosterone and lower norepinephrine, serotonin and cortisol. And, models were specifically formulated for proactive and reactive aggression in that review (Field, 2002). Proactive aggression was hypothesized to feature relatively high dopaminergic function and low norepinephrine and serotonin levels which would normally modulate dopamine. A reactive aggression profile was characterized by high levels of dopamine but also high levels of norepinephrine which would modulate dopamine levels. In the recent literature, violence has not been differentiated as proactive or reactive violence, and no data could be found on EEG or neurotransmitter/neurohormone profiles for violent adolescents. Most of the studies have grouped different types of violent behavior together as an outcome variable rather than specifying different types of violent behavior, e.g. proactive and reactive aggression and physical and relational violence. Some have given specific rates and gender differences for physical and relational violence, but most studies have been categorized as school or dating violence in general.

Surprisingly, very few intervention studies have appeared in this recent literature on adolescent violence. No intervention studies appeared on school violence, although classroom interventions were effective for dating violence. Consistent with our earlier model on violence and touch deprivation in adolescents (Field, 2002), massage therapy has been effective with violent adolescents. In this study, a group of violent adolescents who received massage 
therapy as compared to a group who received relaxation therapy showed more empathy and were rated as being less aggressive and their dopamine levels were lower by the end of the study (Diego, et al, 2002). Unfortunately, no replication studies could be found showing that physical stimulation (i.e. simply moving the skin as in massage therapy) could lower aggression and dopamine levels in violent adolescents. The importance of further intervention research is highlighted by the seriousness and prevalence of school and dating violence. Although this literature has a number of limitations, it has highlighted the importance of continuing theoretical and empirical research on school and dating violence in adolescents as well as interventions to prevent school and dating violence in adolescents.

\section{References}

1. Agbaria, Q. \& Daher, W. (2015). School violence among Arab adolescents in Israel and its relation to self-control skills and social support. Psychology Report, 117, 1-7.

2. Aizpitarte, A., Alonso-Arbiol, I. \& Van de Vijver, F.J.R. (2017a). An explanatory model of dating violence risk factors in Spanish adolescents. Journal of Research on Adolescence, 27, 797809.

3. Aizpitarte, A., Alonso-Arbiol, I., Van de Vijver, F.J.R., Perdomo, M.C., Galvez-Sorbal, J.A. et al. (2017b). Development of a dating violence assessment tool for late adolescence across three countries: The Violence in Adolescents' Dating Relationships Inventory (VADRI). Journal of Interpersonal Violence, 32, 2626-2646.

4. AlBuhairan, F., Abou Abbas, O., El Sayed, D., Badri, M., Alshahri, S. et al. (2017). The relationship of bullying and physical violence to mental health and academic performance: A cross-sectional study among adolescents in Kingdom of Saudi Arabia. International Journal of Pediatrics \& Adolescent Medicine, 4, 61-65.

5. Anand, T., Kishore, J., Grover, S., Bhave, S. \& Yadav, S. (2018). Beliefs supporting violence, attitudes and aggressive behavior among school adolescents in rural Delhi. Community Mental Health Journal, 2018 Aug 4. Doi: 10.1007/s10597018-0315-z. [Epub ahead of print].

6. Baker, C.K. (2017). What role do peers play in adolescent dating? Insights from adolescents with a history of dating violence. Violence Against Women, 23, 178-201.
7. Bala, M.O., Chehab, M.A., Al-Dahshan, A., Saadeh, S. \& Al Khenji, A. (2018). Violence among adolescents in Qatar: Results from global school-based student health survey, 2011. Cureus, 2018 Jul 2;10(7):e2913. Doi: 10.7759/cureus.2913.

8. Ballesteros, M.F., Williams, D.D., Mack, K.A., Simon, T.R. \& Sleet, D.A. (2018). The epidemiology of unintentional and violencerelated injury morbidity and mortality among children and adolescents in the United States. International Journal of Environmental Research and Mental Health, 2018 Mar 28;15(4). Pii: E616. Doi: 10.3390/ijerph 15040616.

9. Bao, P., Jing, J., Yang, W.H., Li, X.H. \& Cai, Y.S. (2016). Violence-related behavior among adolescents and its association with cognitive emotion regulation strategies. World Journal of Pediatrics, 12, 82-87.

10. Barriera, A.K., de Lima, M.L., Bigras, M., Njaine, K. \& Assis, S.G. (2014). Directionality of physical and psychological dating violence among adolescents in Recife, Brazil. Brazilian Journal of Epidemiology, 17, 217-228.

11. Baskin-Sommers, A.R., Waller, R., Fish, A.M. \& Hyde, L.W. (2015). Callous-unemotional trait trajectories interact with earlier conduct problems and executive control to predict violence and substance use among high risk male adolescents. Journal of Abnormal Child Psychology, 43, 15291541.

12. Bell, T.M., Qiao, N., Jenkins, P.C., Siedlecki, C.B. \& Fecher, A.M. (2016). Trends in emergency department visits for nonfatal violence-related injuries among adolescents in the United States, 2009-2013. Journal of Adolescent Health, 58, 573-575.

13. Bond, R.M. \& Bushman, B.J. (2017). The contagious spread of violence among US adolescents through social networks. American Journal of Public Health, 107, 288-294.

14. Buckley, L. \& Chapman, R.L. (2017). Associations between self-reported concussion with later violence injury among Australian early adolescents. Journal of Public Health (Oxf), 39, 52-57.

15. Bundock, K., Chan, C. \& Hewitt, O. (2018). Adolescents' help-seeking behavior and interventions following adolescent dating violence: A systematic review. Trauma, Violence \& Abuse, 2018 Jan 1:1524838018770412. Doi: 10.1177/1524838018770412.[Epub ahead of print].

16. Calvete, E., Orue, I. \& Gamez-Guadix, M. (2015). Reciprocal longitudinal associations between substance use and child-to-parent violence in 
adolescents. Journal of Adolescence, 44, 124133.

17. Courtain, A. \& Glowacz, F. (2018). Exploration of dating and related attitudes among adolescents and emerging adults. Journal of Interpersonal Violence, 2018 Apr 1:886260518770185. Doi: 10.1177/0886260518770185. [Epub ahead of print].

18. Crooks, C.V., Jaffe, P., Dunlop, C., Kerry, A. \& Exner-Cortens, D. (2019). Preventing genderbased violence among adolescents and young adults: Lessons from 25 years of program development and evaluation. Violence Against Women, 25, 29-55.

19. de Oliviera, T.B., Pinto, M.S., de Maacedo, R.F., de Oliviera, T.S. \& Cavalcanti, A.L. (2014). Characterization of morbidity from interpersonal violence in Brazilian children and adolescents. Iran Journal of Public Health, 43, 1184-1191.

20. Diego, M.A., Field, T., Hernandez-Reif, M., Shaw, J., Castellanos, D. \& Rothe, E. (2002). Aggressive adolescents benefit from massage therapy. Adolescence, 37, 597-608.

21. Draucker, C.B., Smith, C., Mazurczyk, J., Thomas, D., Ramirez, P. et al. (2016). Unique outcomes in the narratives of young adults who experienced dating violence as adolescents. Journal of American Psychiatric Nurses Association, 22, 112-121.

22. Dupont-Reyes, M.J., Fry, D., Rickert, V.I., Bell, D.L., Palmetto, N. et al. (2014). Relationship violence, fear, and exposure to youth violence among adolescents in New York City. Journal of Interpersonal Violence, 29, 2325-2350.

23. Farrell, A.D., Mehari, K.R., Kramer-Kuhn, A. \& Goncy, E.A. (2014). The impact of victimization and witnessing violence on physical aggression among high-risk adolescents. Child Development, 85, 1694-1710.

24. Field, T. (1999). American adolescents touch each other less and are more aggressive toward their peers as compared with French adolescents. Adolescence, 34, 753-758.

25. Field, T. (2002). Violence and touch deprivation in adolescents. Adolescence, 37, 735-749.

26. Field, T. (2009). Heartbreak. Indiana: Xlibris.

27. Field, T. (2018a). Internet addiction in adolescents: A review. Journal of Addictions and Therapies, 1, 1-11.

28. Field, T. (2018b). Cyberbullying: A narrative review. Journal of Addiction Therapy and Research, 2, 10-27.

29. Field, T. (2019a). Adolescent depression: A narrative review. Anxiety and Depression.
30. Field, T. (2019b). Suicide ideation and attempts in adolescents: A narrative review. Anxiety and Depression

31. Golshiri, P., Farajzadegan, Z., Tavakoli, A. \& Heidari, K. (2018). Youth violence and related risk factors: A cross-sectional study in 2800 adolescents. Advanced Biomedical Research, 2018 Oct 31;7:138. Doi: 10.4103/abr.abr_137_18. eCollection 2018.

32. Gonzalez-Guarda, R.M., Guerra, J.E., Cummings, A.A., Pino, K. \& Becerra, M.M. (2015). Examining the preliminary efficacy of a dating violence prevention program for Hispanic adolescents. Journal of School Nursing, 31, 411-421.

33. Haglund, K., Belknap, R.A., Edwards, L.M., Tassara, M., Joven, J.V. et al. (2018). The influence of masculinity on male adolescents' perceptions regarding dating relationships and dating violence. Violence Against Women, 2018 Oct 25: 1077801218808395. Doi: 10. 1177/1077801218808395. [Epub ahead of print].

34. Hautala, D.S., Sittner-Hartshorn, K.J., Armenta, B. \& Whitbeck, L. (2017). Prevalence and correlates of physical dating violence among North American indigenous adolescents. Youth \& Society, 49, 295-317.

35. Herbert, A., Gilbert, R., Gonzalez-Izuierdo, A. \& $\mathrm{Li}$, L. (2015). Violence, self-harm and drug or alcohol misuse in adolescents admitted to the hospitals in England for injury: A retrospective cohort study. BMJ Open., 2015 Feb 9; 5(2):e006079. Doi: 10.1136/bmjopen-201400679.

36. Hong Le, M.T., Tran, T.D., Nguyen, H.T. \& Fisher, J. (2014). Early marriage and intimate partner violence among adolescents and young adults in Viet Nam. Journal of Interpersonal Violence, 29, 889-910.

37. Ilie, G., Mann, R.E., Boak, A., Hamilton, H.A., Rehm, J. et al. (2017). Possession of weapon and school violence among adolescents and their association with history of traumatic brain injury, substance use and mental health issues. Injury, 48, 285-292.

38. Johnson, R.M., LaValley, M., Schneider, K.E., Musci, R.J., Pettoruto, K. et al. (2017). Marijuana use and physical dating violence among adolescents and emerging adults: A systematic review and meta-analysis. Drug \& Alcohol Dependence, 174, 47-57.

39. Kelishadi, R., Babaki, A.E., Qorbani, M., Ahadi, Z., Heshmat, R. et al. (2015). Joint association of active and passive smoking with psychiatric distress and violence behaviors in a representative sample of Iranian children and adolescents: the CASPIAN-IV Study. 
International Journal of Behavioral Medicine, 22, 652-661.

40. Khoury-Kassabri, M. \& Attar-Schwartz, S. (2014). Adolescents' reports of physical violence by peers in residential care settings: An ecological examination. Journal of Interpersonal Violence, 29, 659-682.

41. Khubchandani, J. \& Price, J.H. (2018). Violence related behaviors and weapon carrying among Hispanic adolescents: Results from the National Youth Risk Behavior Survey, 2001-2015. Journal of Community Health, 43, 391-399.

42. Khurana, A., Bleakley, A., Ellithorpe, M.E., Hennessy, M., Jamieson, P.E. et al. (2019). Media violence exposure and aggression in adolescents: A risk and resilience perspective. Aggressive Behavior, 45, 70-81.

43. Kim, J.H., Kim, J.Y. \& Kim, S.S. (2016). School violence, depressive symptoms, and help-seeking behavior: A gender-stratified analysis of biethnic adolescents in South Korea. Journal of Preventative Medicine and Public Health, 49, 6168.

44. Kulakci-Altintas, H. \& Ayaz-Alkaya, S. (2019). Parental attitudes perceived by adolescents, and their tendency for violence and affecting factors. Journal of Interpersonal Violence, 34, 200-216.

45. Lindberg, N., Sailas, E. \& Kaltiala-Heino, R. (2012). The copycat phenomenon after two Finnish school shootings: An adolescent psychiatric perspective. BMC Psychiatry, $2012 \mathrm{Jul}$ 28;12:91. Doi: 10.1186/1471-244X-12-91.

46. Loeb, A., Deardorff, J. \& Lahiff, M. (2014). High expectations across multiple domains, peer norms, and physical dating violence among California adolescents. Journal of Interpersonal Violence, 29, 2035-2053.

47. Lundgren, R. \& Amin, A. (2015). Addressing intimate partner and sexual violence among adolescents: Emerging evidence of effectiveness. Journal of Adolescent Health, 56, S42-50.

48. Morelli, M., Bianchi, D., Baiocco, R., Pezzuti, L. \& Chirumbolo, A. (2016). Sexting, psychological distress and dating violence among adolescents and young adults. Psicothema, 28, 137-142.

49. Mrug, S., Madan, A. \& Windle, M. (2016). Emotional desensitization to violence contributes to adolescents' violent behavior. Journal of Abnormal Child Psychology, 44, 75-86.

50. Ohlert, J., Seidler, C., Rau, T., Fegert, J. \& Allroggen, M. (2017). Comparison of psychopathological symptoms in adolescents who experienced sexual violence as a victim and/or as a perpetrator. Journal of Child Sex Abuse, 26, 373-387.
51. Peckins, M.K. \& Susman, E.J. (2015). Variability in diurnal testosterone, exposure to violence, and antisocial behavior in young adolescents, Developmental Psychopathology, 27, 1341-1352.

52. Pollanen, K., de Vries, H., Mathews, C., Schneider, F. \& de Vries, P.J. (2018). Beliefs about sexual intimate partner violence perpetration among adolescents in South Africa. Journal of Interpersonal Violence, 2018 Feb 1:886260518756114.

Doi: 10.1177/0886260518756114. [Epub ahead of print].

53. Pottie, K., Dahal, G., Georgiades, K., Premji, K. \& Hassan, G. (2015). Do first generation immigrant adolescents face higher rates of bullying, violence and suicidal behaviors than do third generation and native born? Journal of Immigrant and Minority Health, 17, 1557-1566.

54. Rao, S., Shah, N., Jawed, N., Inam, S. \& Shafique, K. (2015). Nutritional and lifestyle risk behaviors and their association with mental health and violence among Pakistani adolescents: Results from the national survey of 4583 individuals. BMC Public Health, 2015 Apr 28; 15:431. Doi: 10.1186/s12889-015-1762-x.

55. Russell, M., Cupp, P.K., Jewkes, R.K., Gevers, A. \& Mathews, C. (2014). Intimate partner violence among adolescents in Cape Town, South Africa. Prevention Science, 15, 283-295.

56. Sadeghi, S., Farajzadegen, Z., Kelishadi, R. \& Heidari, K. (2014). Aggression and violence among Iranian adolescents and youth: A 10-year systematic review. International Journal of Preventative Medicine, 5, S83-96.

57. Salas-Wright, C.P., Nelson, E.J., Vaughn, M.G., Reingle-Gonzalez, J.M. \& Cordova, D. (2017). Trends in fighting and violence among adolescents in the United States, 2002-2014. American Journal of Public Health, 107, 977-982.

58. Salas-Wright, C.P., Vaughn, M.G. \& Maynard, B.R. (2014). Religiosity and violence among adolescents in the United States: Findings from the national survey on drug use and health 20062010. Journal of Interpersonal Violence, 29, 11781200. Ciência \& saúde coletiva, 23, 2909-2916.

59. Senanayake, S.J., Gunawardena, S., Wickramasinghe, S., Wickramasinghe, C., Gunawardena, N.S. et al. (2019). Prevalence and correlates of interpersonal violence among inschool adolescents in Sri Lanka: Results from the 2106 Sri Lankan global school-based health survey. Asia Pacific Journal of Public Health, 2019 Jan 24: $1010539519825600 . \quad$ Doi: 10.1177/1010539519825600. [Epub ahead of print]. 
60. Sharma, B., Nam, E.W., Kim, H.Y. \& Kim, J.K. (2016). The influence of witnessing inter-parental violence and bullying victimization in involvement in fighting among adolescents: Evidence from a school-based cross-sectional survey in Peru. Journal of Lifestyle Medicine, 6, 27-35.

61. Silva, B.R.V.S., Silva, A.O.D., Passos, M.H.P.D., Soares, F.C.,Valenca, P.A.M. et al. (2018). Negative self-perceived health associated with school violence in adolescents. Ciencia \& Saude Coletiva, 23, 2909-2916.

62. Singh, V., Epstein-Ngo, Q., Cunningham, R.M., Stoddard, S.A., Chermack, S.T. et al. (2015). Physical dating violence among adolescents and young adults with alcohol misuse. Drug \& Alcohol Dependence, 153, 364-368.

63. Sosa-Rubi, S.G., Saavedra-Avendano, B., Piras, C., Van Buren, S.J. \& Bautista-Arredondo, S. (2017). True love: Effectiveness of a schoolbased program to reduce dating violence among adolescents in Mexico City. Prevention Science, $18,804-817$.
64. Stensland, S.O., Thoresen, S., Wentzel-Larsen, T. \& Dyb, G. (2015). Interpersonal violence and overweight in adolescents: the HUNT Study. Scandinavian Journal of Public Health, 43, 18-26.

65. Swain, S., Mohanan, P., Sanah, N., Sharma, V. \& Ghosh, D. (2014). Risk behaviors related to violence and injury among school-going adolescents in Karnataka, Southern India. International Journal of Adolescent Medical Health, 26, 551-558.

66. Wall Meyers, T.D., Salcedo, A., Frick, P.J., Ray, J.V., Thornton, L.C. et al. (2018). Understanding the link between exposure to violence and aggression in justice-involved adolescents. Developmental Psychopathology, 30, 593-603.

67. Yu, R., Branje, S., Meeus, W., Cowen, P. \& Fazel, S. (2018). Depression, violence and cortisol awakening response: A 3-year longitudinal study in adolescents. Psychological Medicine, $2018 \mathrm{Jul}$ 17:1-8. Doi: 10.1017/S0033291718001654. [Epub .ahead of print]

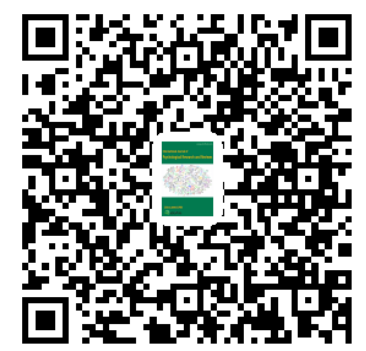

